\title{
The metaphoric nature of the ordinal position effect
}

\author{
Dandan Zhou ${ }^{1,2,3}$, Hanxi Zhong1, 2,3, Wenshan Dong ${ }^{1,2,3}$, Min Li ${ }^{1,2,3}$, Tom \\ Verguts $^{4}$, and Qi Chen ${ }^{1,2,3 *}$ \\ ${ }^{1}$ School of Psychology, South China Normal University, 510631 Guangzhou, China \\ ${ }^{2}$ Center for Studies of Psychological Application, South China Normal University, \\ 510631 Guangzhou, China \\ ${ }^{3}$ Guangdong Key Laboratory of Mental Health and Cognitive Science, South China \\ Normal University, 510631 Guangzhou, China \\ ${ }^{4}$ Department of Psychology, Ghent University, 9000 Ghent, Belgium \\ Running head: Ordinal position effect \\ Correspondence: Qi Chen
}

School of Psychology

South China Normal University

510631 Guangzhou

P.R. China

Email: chen.qi@m.scnu.edu.cn 


\begin{abstract}
Serial orders are thought to be spatially represented in working memory: The beginning items in the memorized sequence are associated with the left side of space and the ending items associated with the right side of space. However, the origin of this ordinal position effect has remained unclear. It was suggested (Guida, et al., 2018) that the reading / writing experience shapes the direction of serial order-space interaction. An alternative hypothesis is that it originates from the "more is right" / “more is up" spatial metaphors we use in daily life (Lakoff \& Johnson, 2003). We can adjudicate between the two theories in Chinese subjects; they read left-to-right, but also have a culturally ancient top-to-bottom reading / writing direction. Thus, the reading / writing theory predicts no or a top-to-bottom effect in serial order-space interaction; whereas the spatial metaphor theory predicts a clear bottom-to-top effect. We designed three experiments to investigate this issue. First, we found a left-to-right ordinal position effect, replicating results obtained in Western populations. However we observed a vertical ordinal position effect in a bottom-to-top direction, which was itself modulated by (left / right) hand positions. We suggest that order-space interactions are a case of metaphoric comprehension, which itself may ground cognitive processing.
\end{abstract}

Keywords: working memory, ordinal position effect, SNARC, metaphor. 
The metaphoric nature of the ordinal position effect

\section{Introduction}

In everyday life, we must maintain information in working memory (WM) for proper cognitive functioning. This high-level cognitive ability appears to be grounded in the sensorimotor system (Barsalou, 2008; Dehaene \& Cohen, 2007). For instance, a series of studies recently observed an association between serial order in WM and external spatial processing (Ginsburg et al., 2017; Guida et al., 2016; Guida and Lavielle-Guida, 2014; van Dijck and Fias, 2011). Subjects responded faster to early items in the memorized sequence when they had to press the left hand; whereas the late items in the memorized sequence were responded to faster when they had to press the right hand. (Abrahamse, van Dijck, \& Fias, 2017; Abrahamse, van Dijck, Majerus, \& Fias, 2014). This phenomenon was termed the ordinal position effect (Ginsburg, van Dijck, Previtali, Fias, \& Gevers, 2014).

Recent studies have started to explore the conditions of the ordinal position effect. Guida et al. (2016) have documented that the ordinal position effect occurred irrespective of whether the remembered verbal items in the sequence were presented in visual or in auditory form. In addition, Ginsburg et al. (2017) also noted that the ordinal position effect is domain-general (e.g., it occurs in the verbal and the visuospatial domains). Furthermore, they found that the ordinal position effect was observed only when the remembered items had a semantic content, and the strength of this effect was significantly related to the semantic content of the to-be-remembered items. So, Ginsburg et al. (2017) suggested that semantic activation of the to-be-remembered items is a necessary condition for the spatial coding of serial order.

Some authors have also discussed the origin of the ordinal position effect in WM. 
Guida, et al. (2018) proposed that its origin relates to the reading / writing direction. Three groups (left-to-right Western readers, right-to-left Arabic readers, and Arabic-speaking illiterates) were tested. They found a left-to-right ordinal position effect for left-to-right Western readers, a right-to-left effect for right-to-left Arabic readers, and no reliable spatial bias for Arabic-speaking illiterates. Guida, et al. (2018) suggested that novel non-spatial information in WM was mentally organized in a culturally determined way.

An alternative theory states that the ordinal position effect is instead due to a general application of "metaphors we live by" (Lakoff \& Johnson, 2003). A careful analysis of everyday speaking and writing led Lakoff and Johnson to conclude that metaphor is pervasive in everyday life, not just in language, but also in thought and action (Lakoff \& Johnson, 2003, p 4). They proposed that human conceptual systems are fundamentally metaphorical in nature. In line with this theory, we suggest that the ordinal position effect might originate from two such spatial metaphors "more is right" and "more is up". There are several occasions in daily life that support such metaphors; for the "more is right" metaphor, we can refer to the pervasive writing of “number lines” from left to right in Western cultures (Rafael E. Núñez, 2011). For the "more is up" metaphor, consider the ubiquitous physical observation that water poured early into a cup will end up at the bottom of that cup; but water poured later ends up at the top. Correspondingly, we suggest that the early items in the memorized sequence associate with the left side, while late items associate with the right side in the horizontal direction ("more is right"). In the vertical direction, the early items associate with the bottom side, while late items associate with the top side ("more is up"). 
The theories can be disentangled in a Chinese population. Chinese usually read and write left to right, but there is also an existing (weaker) top to bottom reading / writing culture (as in Chinese calligraphy, Spring Festival couplets, and so forth). Our experimental procedures were similar to those of Ginsburg et al. (2017) and van Dijck and Fias, (2011), combining a memory task and a classification task. We first attempt to replicate the horizontal ordinal position effect. Both theories predict it in Chinese. However, critically, we then investigate the ordinal position effect in the vertical direction. We suggest that if the effect is related to the reading / writing direction, we would observe a vertical effect aligned in the top-to-bottom direction. If the ordinal position effect instead originates from the "more is up" metaphor, there should be a vertical effect in the bottom-to-top direction.

\section{General Method}

\subsection{Participants}

A total of 96 right-handed undergraduate students from South China Normal University (SCNU) voluntarily participated in this study. All of them were Mandarin Chinese speakers (63 females, mean age 20.75 years, from 18 to 26). They all had normal or corrected-to-normal vision. After the experiment, the participants received modest monetary compensation. The present study was approved by the Human Research Ethics Committee for Non-Clinical Faculties, School of Psychology, South China Normal University.

\subsection{Material}

All the experiments used the same set of stimuli: 4 fruits ("xī guā"-watermelon, “xīang jīao"-banana, "pú táo"-grape, “jú zǐ”-orange) and 4 vegetables (“qíe zi”- 
eggplant, “yáng cōng”-onion, "huáng guā”-cucumber, “wān dòu”-peas) in Chinese characters. These two categories of words were all high frequency, and were selected from the SUBTLEX-CH (Cai \& Brysbaert, 2010). There were no significant differences between them in word frequencies $[t(6)=.17, p>.05]$ or number of strokes $[t(6)=-.45, p>.05]$.

\subsection{Procedure}

The experiments were performed using E-Prime 2 Professional Software (Psychology Software Tools) in a quiet room. The viewing distance was approximately $50 \mathrm{~cm}$ from a 23-inch LCD computer screen with a resolution of $1920 \times 1080$ pixels. For each experiment, the participants had to correctly complete 16 blocks. A new sequence was generated for every block. Each block was divided into three subsequent phases: an encoding phase, a classification phase and a control phase. In all experiments, we only changed the classification phase, the other two phases (encoding phase and control phase) remained the same.

During the encoding phase, 4 words ( 2 fruits and 2 vegetables) forming a sequence were sequentially presented one at a time on the center of the screen. They were written in 35-point Courier New font in bold, in white color on a black background. Participants were first asked to memorize the serial order of words in the order of presentation. When the presented word was successful encoded, participants pressed the "SPACE" button on the keyboard to go to the next word. The words in the sequence successively proceeded from one to the next, centrally on the screen. In order to maximize the chance that the four words were correctly maintained in the order of presentation, following the final word, there was a 2,500 ms blank screen interval for rehearsal. 
After rehearsal, the classification phase started. All words (four memorized words and four other non-memorized words) from the set were randomly presented twice. There was a restriction that two consecutive trials did not contain the same word.

Participants were instructed to classify the presented word as fruit or vegetable, and only respond to the memorized words from the encoding phase (go/no-go paradigm). Each trial started with a fixation cross in the center of the screen. After $500 \mathrm{~ms}$, a word appeared centrally. If this word belonged to the memorized sequence, participants had to quickly and accurately judge it as fruit or vegetable. The word appeared until the participant made a motor response or the response deadline (1500 ms) was reached, followed by an inter-trial interval with a blank screen for $1000 \mathrm{~ms}$. Then the next trial was initiated. The speed and accuracy of motor responses were collected via button presses on the keyboard.

The last phase (control phase) was designed to make sure that participants had maintained the memorized sequence in the correct order. Three pairs of words were randomly selected from the memorized sequence and presented successively on the screen. One word of the pair was presented on the right of the screen, the other on the left. The two words were always neighboring items in the memorized sequence. For each pair, participants were required to indicate if the left word was presented before or after the right word in the sequence. If the left word was presented before the right word, they had to press the "O" button; if it was not, they had to press the "N" button. There was no time limit for responding. As long as there was one mistaken response, the given block was introduced again after this control phase. In the experiment, we only took into account the blocks with a $100 \%$ correct control phase.

\subsection{Data Analysis}


For each participant, the go-trials with incorrect responses and RTs more than 2 SDs from the individual mean were removed from further analysis. The mean RTs of the remaining trials were calculated for each experimental condition. Following Ginsburg et al. (2017), we first applied a repeated measures ANOVA with the variables ordinal position in WM (4: 1-4) and response side (2: left vs. right, or bottom vs. top). A significant interaction between ordinal position and response side means that the ordinal position effect is observed. We also applied a different method to test the ordinal position effect, namely the linear regression approach (Fias, Brysbaert, Geypens, d'Ydewalle, 1996). For each position in WM (from 1 to 4), we compute the difference in RT (dRT: right minus left spatial location, or top minus bottom spatial location). The dRTs were entered in a regression analysis for each participant separately, with position as predictor. A negative regression slope indicates that the early items in the memorized sequence are responded to faster with the left or bottom side; and the late items are responded to faster with the right or top side, which suggests that there is a left-to-right ordinal position effect in the horizontal direction or a bottom-to-top ordinal position effect in the vertical direction. Then we performed an independent samples $t$-test to evaluate whether the regression weights of the group deviated significantly from zero. Finally, we performed a Bayesian one sample $t$-test on the regression slopes to investigate the presence of the effect (Eidswick, 2012; Wagenmakers, Wetzels, Borsboom, \& van der Maas, 2011).

\section{Experiment 1}

\subsection{Method}

31 subjects participated in Experiment 1 ( 27 females, mean age of 21.26). In the classification phase, we asked participants to horizontally place their left index finger 
on the ' $\mathrm{D}$ ' key (left side of space) and the right index finger on the ' $L$ ' key (right side of space). Half of the participants pressed the left button for 'fruits' and the right button for 'vegetables', whereas the other half received the opposite mapping.

\subsection{Results and Discussion}

Trials with incorrect responses $(5.8 \%)$ and RTs more than 2 SDs from the individual mean $(4.5 \%)$ were removed from analysis. One subject with more than $2 S D$ s above the mean errors was excluded. Participants accomplished an average 17.17 blocks (SD $=1.77$ ) before they correctly performed 16 blocks. The average RT of the classification phase was $838.03 \mathrm{~ms}(S D=210.19 \mathrm{~ms})$.

The RTs were entered in a 4 (WM position: $1 / 2 / 3 / 4) \times 2$ (response side: left/right) repeated measures ANOVA. There was a main effect of WM position, $F(3,84)=$ 7.655, $p<.001, \eta^{2}{ }_{p}=.22$. The RTs of each position were as follows: $822,852,861$, $863 \mathrm{~ms}$, which increased gradually. A polynomial contrast confirmed this linear trend $\left[F(1,28)=14.41, p=.001, \eta^{2}=.34\right]$. The interaction between $\mathrm{WM}$ position and response side was significant, $F(3,84)=13.81, p<.001, \eta_{\mathrm{p}}^{2}=.33$. We then used the linear regression approach to further investigate this interaction, and found a significant result [slope $=-40.58 ; t(28)=-5.98, S D=36.53, p<.001]$ (Figure 1a). A Bayes-factor $\left(\mathrm{BF}_{10}\right)$ of 9707.42 strongly supports the presence of the ordinal position effect.

The results of Experiment 1 confirmed the presence of a horizontal ordinal position effect in Chinese subjects, as found in previous studies with Western participants (Ginsburg et al., 2017; Guida, et al., 2018).

\section{Experiment 2}


Given the replication of the horizontal ordinal position effect in Experiment 1 in a Chinese population, we could now turn to our main focus and investigate the ordinal position effect in the vertical direction.

\subsection{Method}

32 new subjects participated in Experiment 2 (23 females, mean age of 20.28). In the classification phase, as done in previous studies (Shaki \& Fischer, 2012) we rotated the keyboard by 90 degree counterclockwise. All participants were instructed to vertically align the left index finger on the " $D$ " key (bottom side of space) and right index finger on the "L" key (top side of space). Half of the participants pressed the bottom key for 'fruits' and the top key for 'vegetables', whereas the other half pressed the top key for 'fruits' and the bottom key for 'vegetables'.

\subsection{Results and Discussion}

Trials with incorrect responses (7.5\%) and RTs more than 2 SDs from the individual mean $(5.0 \%)$ were removed from analysis. Three subject with more than 2 SDs above the mean errors were excluded. Participants accomplished an average 17.45 blocks $(S D=1.86)$ before they correctly performed 16 blocks. The average RT of the classification phase was $856.93 \mathrm{~ms}(S D=217.28 \mathrm{~ms})$.

The 4 (WM position: 1/2/3/4) $\times 2$ (response side: bottom/top) repeated measures ANOVA indicated that there was a main effect of $\mathrm{WM}$ position, $F(3,84)=5.74, p$ $=.001, \eta^{2} \mathrm{p}=.17$. The RTs of each position were as follows: $844,871,866,881 \mathrm{~ms}$, which increased gradually. A polynomial contrast confirmed this linear trend, $F(1,28)$ $=14.54, p=.001, \eta_{\mathrm{p}}^{2}=.34$. Most importantly, the interaction between WM position and response side was significant, $F(3,84)=10.10, p<.001, \eta^{2}=.27$. The linear 
regression approach found there was a significant effect [slope $=-34.30 ; t(24)=$ $-4.771, S D=38.72, p<.001$ ] (Figure $1 b$ ). $\mathrm{A} \mathrm{BF}_{10}$ of 472.71 strongly supports the presence of the vertical ordinal position effect.

Experiment 2 clearly showed a vertical ordinal position effect in Chinese subjects. In line with the metaphor theory, the effect aligned in the bottom-to-top direction. During the classification phase of Experiment 2, subjects vertically aligned their left hand on the bottom side and their right hand on the top side throughout the entire experiment. In principle, two factors could be responsible for this ordinal position effect. One is the "more is up" metaphor we discussed. Alternatively, however, the fact that the right hand was located on the top side might have driven the effect. From this perspective, the effect would still be due to a left-to-right factor. To differentiate between these possibilities, we conducted Experiment 3, where we investigate the vertical ordinal position effect again, but reversed the hand-key assignment of Experiment 2.

\section{Experiment 3}

In Experiment 3, the left hand is placed on the top side and the right hand on the bottom side. If the vertical ordinal position effect is related to the response hands (and thus to a left-to-right alignment), the bottom-to-top effect observed in Experiment 2 would be reversed. Instead, if the ordinal position was due to the spatial "more is up" metaphor, it would be unchanged. Finally, if the effect is reduced relative to Experiment 2, there is evidence that both factors play a role.

\subsection{Method}

33 new subjects participated in Experiment 3 ( 23 females, mean age of 20.60). The classification phase was identical to Experiment 2, except for the hand-key mapping. 
All participants were asked to place their left index finger on the "L" key (top side of space) and right index finger on the "D" key (bottom side of space) to respond. Half of the participants pressed the top key for 'fruits' and the bottom key for 'vegetables', whereas the other half received the opposite response mapping.

\subsection{Results and Discussion}

Trials with incorrect responses $(8.3 \%)$ and RTs more than 2 SDs from the individual mean $(4.6 \%)$ were removed from analysis. One subject with more than 2 SDs above the mean errors was excluded. Participants accomplished an average 17.63 blocks (SD $=1.56)$. The average RT of the classification phase was $882.24 \mathrm{~ms}(S D=221.46 \mathrm{~ms})$.

The statistical analyses were identical to the previous experiments. A significant main effect of WM position was found, $F(3,93)=8.73, p<.001, \eta^{2}{ }_{p}=.22$. The RTs of each position were as follows: 858, 889, 897, $913 \mathrm{~ms}$, which increased gradually. A polynomial contrast confirmed this linear trend $\left[F(1,31)=21.51, p<.001, \eta_{\mathrm{p}}^{2}=.41\right]$. However, the interaction between WM position and response side was not significant, $F(3,93)=1.16, p=.33, \eta^{2}=.04$. We then used the linear regression approach to further investigate this interaction. Although the slope was negative, like in Experiment 2, there was no significant effect [slope $=-2.48 ; t(31)=-.29, S D=48.40$, $p=.77$ ] (Figure 1c). $\mathrm{A} \mathrm{BF}_{10}$ of 0.20 also does not supports the presence of the vertical ordinal position effect in this particular condition. Thus, both hands (by definition left -to-right oriented) and vertical location seem to play a role in the vertical ordinal position effect.

\section{General Discussion}

The purpose of the present study was to investigate the origin of the ordinal position 
effect. We designed three experiments to explore this issue in Chinese readers. In Experiment 1, we replicated the horizontal ordinal position effect, which aligned in the left-to-right direction. The subsequent experiments were conducted to investigate the core issue. In Experiment 2, we found the vertical ordinal position effect in the bottom-to-top orientation, which suggested that the early items in the memorized sequence were associated with the bottom side, while the late items are associated with the top side. In this experiment, the left hand was placed on the bottom side and the right hand on the top side. Although this design reduced the interference from the counterbalancing of hand-key assignment (Shaki \& Fischer, 2012), the procedure might confound the spatial location (top or bottom) with the response hands. We therefore designed Experiment 3, in which the left hand was placed on the top side and the right hand on the bottom side. We found that although the effect was not significant, the serial order-space interaction still aligned in the bottom-to-top direction, suggesting that both horizontal and vertical alignments play a role.

The left-to-right ordinal position effect was previously explained by the reading / writing direction theory (Guida et al., 2018), which suggests that the direction of serial order-space interaction is related to the reading/writing direction. In line with this view, the mental whiteboard hypothesis (Abrahamse et al., 2017, 2014) suggests that the storage and retrieval processes of serial order in WM are based on the internal spatial attention system (Abrahamse et al., 2014). The brain might generate an internal spatial template to "write down" the perceived items, and capture item sequence in WM just like writing the serial items on a sheet of paper (Abrahamse et al., 2017). This theory suggests that reading / writing experience shapes the direction of "writing" on the internal spatial template (Abrahamse et al., 2017, 2014). However, the reading / writing direction hypothesis cannot explain the findings of the bottom-to-top ordinal 
position effect in Chinese subjects, who still retain the top-to-bottom reading / writing culture. Finally, the reading / writing hypothesis cannot explain why the serial order-space interaction was influenced by the spatial location of response hands.

We suggest that the metaphor theory can account for these results. In the horizontal direction, a "more is right" metaphor may drive the ordinal position effect. For the vertical dimension, the effect mainly originates from the "more is up" metaphor (Lakoff \& Johnson, 2003). When the left hand was assigned with the bottom side and the right hand with the top side (In Experiment 2), there were no conflicts between the early-left and early-bottom associations, or between late-right and late-top associations. However, when the hand-key assignment was reversed, the left hand was assigned with the top side and the right hand with the bottom side. We suggest that the left-early associations worked against the top-late associations, and right-late associations against the bottom-early associations. This might contribute to the lack of vertical ordinal position effect in Experiment 3. This spatial metaphor (Lakoff \& Johnson, 2003) is also compatible with the mental whiteboard hypothesis (Abrahamse et al., 2017, 2014); spatial metaphors may drive the directionality of use of the mental whiteboard. However, it remains to be shown how the metaphor theory can explain the "reversed" right / left directionality of the ordinal position effect observed by Guida et al. (2018). We suggest that because of the strong influence of the reading / writing direction, right-to-left readers develop a more dominant "left is more" metaphor. Although this is of course post hoc and remains to be empirically investigated, it allows for a potential reconciliation of the two theories, where the reading / writing direction is just one potential generator of metaphors that guide our actions. 
Metaphoric comprehension has been proposed to describe another spatial-association phenomenon (Winter \& Teenie, 2013), the SNARC (Spatial-Numerical Associations of Response Code) effect (Dehaene, Bossini, \& Giraux, 1993). This reflects the tendency that the small numbers are responded to faster with the left side while large numbers are responded to faster with the right side (Göbel, Shaki, \& Fischer, 2011; Hartmann, Grabherr, \& Mast, 2012; Zebian, 2005). For the horizontal SNARC effect, Winter and Teenie (2013) proposed that the "more is right" metaphor may explain how numbers are represented in the horizontal direction. In addition to the horizontal spatial-numerical associations, several studies have investigated it in the vertical direction (Gevers, Lammertyn, Notebaert, Verguts, \& Fias, 2006; Hartmann, Gashaj, Stahnke, \& Mast, 2014; Hung, Hung, Tzeng, \& Wu, 2008; Ito \& Hatta, 2004; Mengxia Li, Entao Zhang, Yanjun Zhang, Xi Fanga, \& Qiwei Li, 2017; Schwarz \& Keus, 2004; Sell \& Kaschak, 2012; Shaki \& Fischer, 2012). Most studies of the vertical SNARC effect have indicated that small numbers are associated with the bottom side of space, and large numbers with the top (Hartmann et al., 2012; Ito \& Hatta, 2004; Schwarz \& Keus, 2004; Sell \& Kaschak, 2012; Winter \& Teenie, 2013). The researchers put forward the "more is up" spatial metaphors (Gevers et al., 2006; Hartmann et al., 2012; Sell \& Kaschak, 2012; Shaki \& Fischer, 2012; Winter \& Teenie, 2013). We suggest that all these spatial associations are special cases of metaphoric comprehension; and that more generally, space, order, and number interactions derive from metaphoric, culturally determined comprehension (Lakoff \& Johnson, 2003). 
Ordinal position effect 16

\section{Acknowledgments}

This study was supported by grants from the Natural Science Foundation of China (Grants 31671135 and 31300834 to Qi Chen) and the Guangdong Province Universities and Colleges Pearl River Scholar Funded Scheme (2016) to Qi Chen. 


\section{References}

Abrahamse, E. L., van Dijck, J.-P., \& Fias, W. (2017). Grounding Verbal Working Memory: The Case of Serial Order. Current Directions in Psychological Science, 26(5), 429-433. https://doi.org/10.1177/0963721417704404

Abrahamse, E. L., van Dijck, J.-P., Majerus, S., \& Fias, W. (2014). Finding the answer in space: the mental whiteboard hypothesis on serial order in working memory. Frontiers in Human Neuroscience, 8(November), 1-12. https://doi.org/10.3389/fnhum.2014.00932

Barsalou, L. W. (2008). Grounded Cognition. Annual Review of Psychology, 59(1), 617-645. https://doi.org/10.1146/annurev.psych.59.103006.093639

Cai, Q., \& Brysbaert, M. (2010). SUBTLEX-CH: Chinese Word and Character Frequencies Based on Film Subtitles. PLoS ONE, 5(6), e10729. https://doi.org/10.1371/journal.pone.0010729

Dehaene, S., Bossini, S., \& Giraux, P. (1993). The mental representation of parity and number magnitude. Journal of Experimental Psychology: General, 122(3), 371396. https://doi.org/10.1037/0096-3445.122.3.371

Dehaene, S., \& Cohen, L. (2007). Cultural recycling of cortical maps. Neuron, 56(2), 384-398. https://doi.org/10.1016/j.neuron.2007.10.004

Eidswick, J. (2012). A Bayesian alternative to null hypothesis significance testing. Shiken Research Bulletin, 16(1), 2-14. Retrieved from http://www.academia.edu/download/30928452/Eidswick_Bayesian.pdf

Fias, Wim; Brysbaert, Marc; Geypens, Frank; d'Ydewalle, G. (1996). The Importance of Magnitude Information in Numerical Processing: Evidence from the SNARC Effect. Mathematical Cognition, 2(1), 95-110.

https://doi.org/10.1080/135467996387552 
Gevers, W., Lammertyn, J., Notebaert, W., Verguts, T., \& Fias, W. (2006). Automatic response activation of implicit spatial information: Evidence from the SNARC effect. Acta Psychologica, 122(3), 221-233. https://doi.org/10.1016/j.actpsy.2005.11.004

Ginsburg, V., Archambeau, K., van Dijck, J.-P., Chetail, F., \& Gevers, W. (2017). Coding of serial order in verbal, visual and spatial working memory. Journal of Experimental Psychology: General, 146(5), 632-650. https://doi.org/10.1037/xge0000278

Ginsburg, V., van Dijck, J.-P., Previtali, P., Fias, W., \& Gevers, W. (2014). The impact of verbal working memory on number-space associations. Journal of Experimental Psychology: Learning, Memory, and Cognition, 40(4), 976-986. https://doi.org/10.1037/a0036378

Göbel, S. M., Shaki, S., \& Fischer, M. H. (2011). The Cultural Number Line: A Review of Cultural and Linguistic Influences on the Development of Number Processing. Journal of Cross-Cultural Psychology, 42(4), 543-565. https://doi.org/10.1177/0022022111406251

Guida, A., \& Lavielle-Guida, M. (2014). 2011 space odyssey: spatialization as a mechanism to code order allows a close encounter between memory expertise and classic immediate memory studies. Frontiers in Psychology, 5(June), 1-5. https://doi.org/10.3389/fpsyg.2014.00573

Guida, A., Leroux, A., Lavielle-Guida, M., \& Noël, Y. (2016). A SPoARC in the Dark: Spatialization in Verbal Immediate Memory. Cognitive Science, 40(8), 2108-2121. https://doi.org/10.1111/cogs.12316

Guida, A., Megreya, A. M., Lavielle-Guida, M., Noël, Y., Mathy, F., van Dijck, J.-P., \& Abrahamse, E. (2018). Spatialization in working memory is related to literacy 
and reading direction: Culture "literarily" directs our thoughts. Cognition, 175(July 2017), 96-100. https://doi.org/10.1016/j.cognition.2018.02.013

Hartmann, M., Gashaj, V., Stahnke, A., \& Mast, F. W. (2014). There is more than "more is up": Hand and foot responses reverse the vertical association of number magnitudes. Journal of Experimental Psychology: Human Perception and Performance, 40(4), 1401-1414. https://doi.org/10.1037/a0036686

Hartmann, M., Grabherr, L., \& Mast, F. W. (2012). Moving along the mental number line: Interactions between whole-body motion and numerical cognition. Journal of Experimental Psychology: Human Perception and Performance, 38(6), 14161427. https://doi.org/10.1037/a0026706

Hung, Y., Hung, D. L., Tzeng, O. J.-L., \& Wu, D. H. (2008). Flexible spatial mapping of different notations of numbers in Chinese readers. Cognition, 106(3), 14411450. https://doi.org/10.1016/j.cognition.2007.04.017

Ito, Y., \& Hatta, T. (2004). Spatial structure of quantitative representation of numbers: Evidence from the SNARC effect. Memory and Cognition, 32(4), 662-673. https://doi.org/10.3758/BF03195857

Lakoff, G., \& Johnson, M. (2003). George Lakoff and Mark Johnsen ( 2003 ) Metaphors we live by. London: The university of Chicago press . Noter om layout : Innholdsfortegnelse $i$ Word : Journal of Philosophy.

Mengxia Li, Entao Zhang, Yanjun Zhang, Xi Fanga, \& Qiwei Li. (2017). Flexible Verbal-Spatial Mapping in the Horizontal and Vertical SNARC Effects of Mainland Chinese Readers. The American Journal of Psychology, 130(3), 339. https://doi.org/10.5406/amerjpsyc.130.3.0339

Rafael E. Núñez. (2011). No Innate Number Line in the Human Brain. Journal of Cross-Cultural Psychology, 42(4), 651-668. 
https://doi.org/10.1177/0022022111406097

Schwarz, W., \& Keus, I. M. (2004). Moving the eyes along the mental number line: Comparing SNARC effects with saccadic and manual responses. Perception and Psychophysics, 66(4), 651-664. https://doi.org/10.3758/BF03194909

Sell, A. J., \& Kaschak, M. P. (2012). The comprehension of sentences involving quantity information affects responses on the up-down axis. Psychonomic Bulletin \& Review, 19(4), 708-714. https://doi.org/10.3758/s13423-012-0263-5

Shaki, S., \& Fischer, M. H. (2012). Multiple spatial mappings in numerical cognition. Journal of Experimental Psychology: Human Perception and Performance, 38(3), 804-809. https://doi.org/10.1037/a0027562

van Dijck, J.-P., \& Fias, W. (2011). A working memory account for spatial-numerical associations. Cognition, 119(1), 114-119. https://doi.org/10.1016/j.cognition.2010.12.013

Wagenmakers, E., Wetzels, R., Borsboom, D., \& van der Maas, H. L. J. (2011). Why psychologists must change the way they analyze their data: The case of psi: Comment on Bem (2011). Journal of Personality and Social Psychology, 100(3), 426-432. https://doi.org/10.1037/a0022790

Winter, B., \& Teenie, M. (2013). More is up and right : Random number generation along two axes ., (January).

Zebian, S. (2005). Linkages between Number Concepts, Spatial Thinking, and Directionality of Writing: The SNARC Effect and the REVERSE SNARC Effect in English and Arabic Monoliterates, Biliterates, and Illiterate Arabic Speakers. Journal of Cognition and Culture, 5(1), 165-190. https://doi.org/10.1163/1568537054068660 
Ordinal position effect 21

\section{Figure legends}

Figure 1. The ordinal position effect of experiments. The difference in RT (dRT) as a function of position in the working memory (WM) sequence. (a) Experiment 1. The dRTs were obtained by subtracting the average left-hand RTs from the average right-hand RTs. (b) Experiment 2. (c) Experiment 3. The dRTs in Experiment 2 and Experiment 3 were obtained by subtracting the bottom-side RTs from the top-side RTs. 
Figure 1.
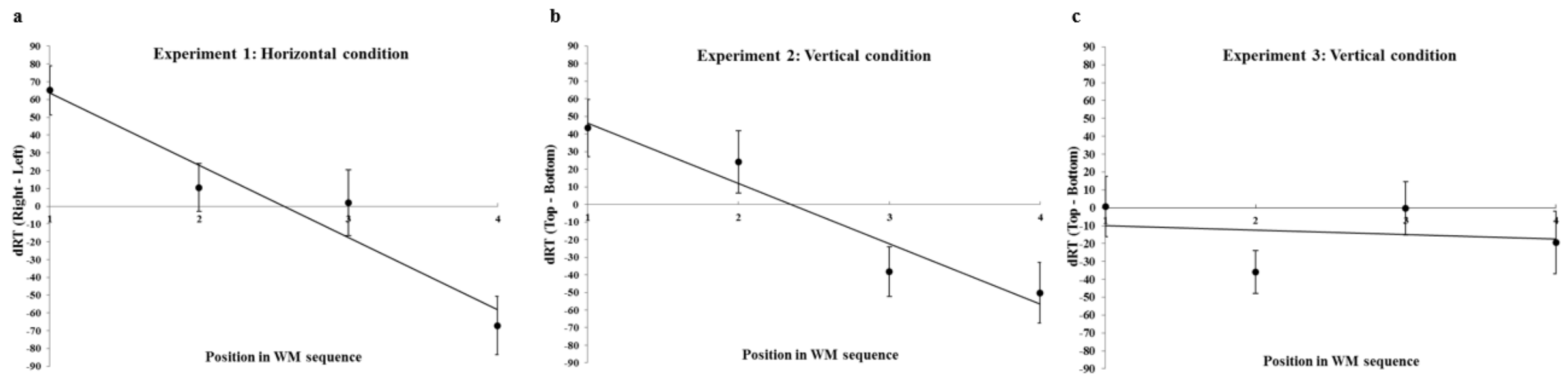